\title{
Correlation between Shear Wave Velocity and Porosity in Porous Solids and Rocks
}

\author{
J. Kováčik ${ }^{1}$ and Š. Emmer ${ }^{2}$ \\ ${ }^{1}$ Institute of Materials \& Machine Mechanics, Slovak Academy of Sciences, Račianska 75, SK-831 02 Bratislava 3, Slovakia \\ ${ }^{2}$ Slovak University of Technology, IVMA STU, Institute of Technologies \& Materials STU, Pionierska 15, \\ SK-831 02 Bratislava 3, Slovakia
}

Correspondence should be addressed to J. Kováčik, ummsjk@savba.sk

Received 6 July 2012; Revised 16 October 2012; Accepted 30 October 2012

Academic Editor: Chengfeng Li

Copyright (C) 2013 J. Kováčik and Š. Emmer. This is an open access article distributed under the Creative Commons Attribution License, which permits unrestricted use, distribution, and reproduction in any medium, provided the original work is properly cited.

\begin{abstract}
The shear wave velocity dependence on porosity was modelled using percolation theory model for the shear modulus porosity dependence. The obtained model is not a power law dependence (no simple scaling with porosity), but a more complex equation. Control parameters of this equation are shear wave velocity of bulk solid, percolation threshold of the material and the characteristic power law exponent for shear modulus porosity dependence. This model is suitable for all porous materials, mortars and porous rocks filled with liquid or gas. In the case of pores filled with gas the model can be further simplified: The term for the ratio of the gas density to the density of solid material can be omitted in the denominator (the ratio is usually in the range of $\left(10^{-4}, 10^{-3}\right)$ for all solids). This simplified equation was then tested on the experimental data set for porous $\mathrm{ZnO}$ filled with air. Due to lack of reasonable data the scientists are encouraged to test the validity of proposed model using their experimental data.
\end{abstract}

\section{Introduction}

The porous materials are usually prepared by various powder metallurgy methods from powders, which composition, particle size, and shape can vary significantly. During the powder consolidation different porosity can be achieved by varying of the technological parameters: such as temperature, external pressure, or time. Compacting starts from just touching powder particles and goes to the lower porosity by the creation and growth of the necks between particles. The subsequent closure of the pore channels leads to the elimination of the pores. Three various porosity ranges can be usually identified, for example, for sintered iron the following porosity ranges can be observed ([1] and references therein):

(i) porosity $<3 \%$ : fully isolated pores of nearly spherical or elliptical shape,

(ii) porosity $>20 \%$ : fully interconnected pores of complex shape, and (iii) porosity between $3 \%$ and 20\%: both isolated and interconnected pores are present in various amounts.

This indicates that the powder consolidation is in general a connectivity problem, which is studied by the percolation theory [2]. According to the percolation theory a critical volume fraction exists, called a percolation threshold, at which a solid phase forms a continual network spanning across the whole system. Below this threshold it is just a pile of powders or mud of powders mixed with liquid. At and above the percolation threshold $p_{c}$ the geometrical, physical, and mechanical properties of the system behave in the form of the power law dependence $\propto\left(\left(p_{c}-p\right) / p_{c}\right)^{t}$ for $p \leq p_{c}$, where $p$ is the porosity within the solid material and $t$ is the critical exponent for the investigated property.

The percolation theory expects that the values of the critical exponents are universal, that is, they do not depend on the structure and geometrical properties of the system, but only depends on the dimension of the problem [2]. On the other hand, the value of the percolation threshold depends significantly on the structure. 


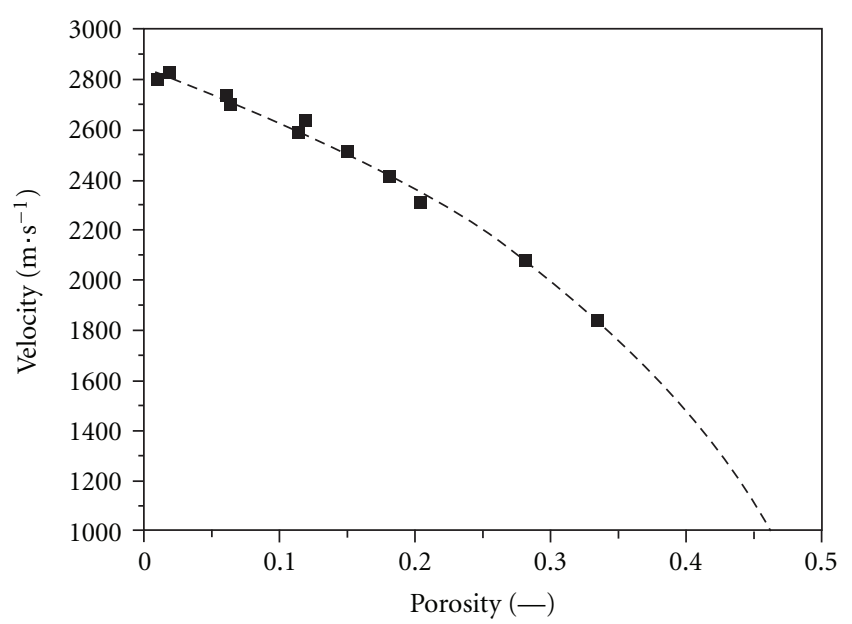

FIGURE 1: Shear wave velocity dependence on porosity for porous $\mathrm{ZnO}[3]$.

TABLE 1: Fitting results for shear wave velocity dependence on porosity for porous $\mathrm{ZnO}$ [3]. There $\chi^{2}$ is a characteristic value of the nonlinear fitting.

\begin{tabular}{lcccc}
\hline $\begin{array}{l}\text { Porosity range } \\
{[-]}\end{array}$ & $\begin{array}{c}c_{S 0} \\
{\left[\mathrm{~ms}^{-1}\right]}\end{array}$ & $\begin{array}{c}p_{c} \\
{[-]}\end{array}$ & $\begin{array}{c}f_{G} \\
{[-]}\end{array}$ & $\begin{array}{c}\chi^{2} \\
{[-]}\end{array}$ \\
\hline $0-0.33$ & $2844 \pm 16$ & $0.52 \pm 0.04$ & $1.22 \pm 0.14$ & 611 \\
\hline
\end{tabular}

Today nondestructive method of the determination of mechanical properties of porous solids is usually required instead of destructive tensile test and/or bending test. Acoustic or ultrasonic measurements are often used in this case [4, 5]. Measurements are usually conducted at sonic $(20-50 \mathrm{kHz})$ and ultrasonic $(300-700 \mathrm{kHz})$ frequencies using pulse transmission technique. The longitudinal and shear wave velocities are then determined and used to calculate the mechanical properties of the porous materials.

The aim of this paper is to model the dependence of the shear wave velocity on the porosity for porous solids and rocks.

\section{Theoretical}

The shear wave velocity, $c_{S}$, depends on the following material properties [2]:

$$
c_{S}=\sqrt{\frac{G}{\rho}}
$$

where $G$ is the shear modulus and $\rho$ is the density of the material.

Recently [1, 6-8], in the case of homogeneous isotropic porous materials, modulus of elasticity $E$, shear modulus $G$, and Poisson's ratio $v$ were modelled using power law dependence on porosity on the basis of the percolation theory using the idea of percolation threshold $[2,9,10]$. As was mentioned above, the percolation threshold is a porosity at which the mechanical properties of porous material become zero (the porous material does not exist anymore as entity). It was further showed $[1,6]$ how the powder size, preparation method (various amounts of sintering additives), the powder shape, and the investigated porosity range influence the values of characteristic exponent and percolation threshold for elastic modulus and shear modulus of porous materials prepared by powder metallurgy.

According to percolation theory the shear modulus porosity dependence is expressed as follows [6]:

$$
G=G_{0}\left(\frac{p_{c}-p}{p_{c}}\right)^{f_{G}} \text { for } p \leq p_{c}
$$

where $G$ is the effective shear modulus of porous material with porosity $p, G_{0}$ is shear modulus of solid material, $p_{c}$ is a percolation threshold, and $f_{G}$ is the characteristic exponent for the shear modulus of porous material.

The effective density of porous solid is also dependent on porosity. This dependence can be simply expressed by the rule of mixture as follows:

$$
\rho=\rho_{0} \cdot(1-p)+\rho_{f} \cdot p,
$$

where $\rho_{0}$ is the density of the solid material and $\rho_{f}$ is the density of pore filling material (gas, liquid) at given temperature and pressure.

Now, (2) and (3) can be put into (1) thus obtaining

$$
c_{S}=c_{S 0} \sqrt{\frac{\left(\left(p_{c}-p\right) / p_{c}\right)^{f_{G}}}{1-p+\left(\rho_{f} / \rho_{0} \cdot\right) \cdot p}} \text { for } p \leq p_{c}
$$

where $c_{S 0}$ is the shear wave velocity of bulk material.

In the case when the porous solid is filled with gas or air the density of air/gas is always significantly smaller (e.g., at $20^{\circ} \mathrm{C}$ and $101.325 \mathrm{kPa}$, dry air has a density of $1.2041 \mathrm{~kg} \cdot \mathrm{m}^{-3}$ [11]) in comparison to the density of usual solid materials. Therefore, the term containing the density of air/gas in (4) can be neglected. Thus, the proposed equation can be simplified in the following way:

$$
c_{S}=c_{S 0} \sqrt{\frac{\left(\left(p_{c}-p\right) / p_{c}\right)^{f_{G}}}{1-p}} \text { for } p \leq p_{c}
$$

\section{Experimental Results and Discussion}

To prove the hypothesis it is necessary to do experiments or to use already published data. Unfortunately the "proper" experimental data (almost the same technology of the preparation, as wide as possible investigated porosity range, data also in the vicinity of the percolation threshold) is very hard to find over the scientific journals for the shear wave velocity of porous materials filled with air/gas. To authors' knowledge, all these requirements were fulfilled only for the experimental data for porous $\mathrm{ZnO}$ [3]. Therefore, the proposed equation was tested only using the experimental data of porous $\mathrm{ZnO}$ [3], what is also the main lack of this article. As Table 1 and Figure 1 indicate (5) describes the investigated experimental data relatively well. Besides determination of the value of 
percolation threshold for porous $\mathrm{ZnO}$ to $0.52 \pm 0.04$ it also estimates the velocity of shear waves in solid $\mathrm{ZnO}$ to $2844 \pm 16 \mathrm{~ms}^{-1}$ and the characteristic exponent $f_{G} 1.22$ \pm 0.14 . The percolation threshold value coincides with the percolation threshold value obtained for modulus of elasticity and shear modulus of $\mathrm{ZnO}[1,6]: 0.51 \pm 0.06$ and $0.51 \pm$ 0.04 , respectively. The shear wave velocity of solid $\mathrm{ZnO}$ is comparable to the value of $2810 \mathrm{~ms}^{-1}$ for solid material in the scientific literature [3]. The value of the characteristic exponent $f_{G}$ is higher than the corresponding value of 1.18 \pm 0.15 determined from the percolation model for shear modulus [6].

\section{Conclusions}

Summarising, the model proposed on the basis of the percolation theory enables to describe and explain the complex shear wave velocity dependence on porosity of porous $\mathrm{ZnO}$ using only 3-model parameters: shear wave velocity of the solid material, percolation threshold, and power law characteristic exponent for shear modulus. However, further testing of the model is required to prove its validity for other porous ceramics and solids. It is expected that all model parameters will vary due to the preparation method, the powder size, powder shape, and the investigated porosity range influence.

It is evident that the shear wave velocity dependence is more complex function of porosity in comparison with simple power law scaling of Young's and shear modulus with porosity. Moreover it indicates that not all properties of the porous material ought to fulfil the simple scaling power law dependence of the investigated mechanical property on porosity.

It must be further noted, that one can start with the shear wave velocity model in the scaling form identical with (2), but after putting this formula into the (1) and accepting (3) the shear modulus dependence on porosity ought to become more complex. However, this is in contradiction with the generally accepted theoretical results and also with the observed experimental results.

Therefore, the scientists are encouraged to test the validity of proposed model using their experimental data.

\section{Appendix}

There is a lot of data across literature about mechanical properties of porous solids, but only small part of them deal with shear wave velocities and most of them did not fulfil the basic requirements for reasonable fitting to any model, such as: Almost the same technology of the preparation, as wide as possible investigated porosity range, and data also in the vicinity of the percolation threshold. To be honest most of the data in the literature are from narrow porosity range where simple linear regression is more precise as any model.

For this reason we have used only the data for porous $\mathrm{ZnO}$ as they were the only one we were able to find in the literature and which approximately fulfilled requirements for reasonable fitting. Therefore we write this paper, to encourage other scientists to create proper data set and to test the validity of the proposed model.

\section{Acknowledgment}

The authors would like to express their gratitude to Slovak Government for funding of this work within following projects: APVV-20-057805, APVV-VMSPII-0009-09, VEGA 2/0191/10, VEGA 1/0189/12.

\section{References}

[1] J. Kováčik, "Correlation between Young's modulus and porosity in porous materials," Journal of Materials Science Letters, vol. 18, no. 13, pp. 1007-1010, 1999.

[2] D. Stauffer and A. Aharony, Introduction to Percolation Theory, Taylor \& Francis, London, 2nd edition, 1992.

[3] L. P. Martin, D. Dadon, and M. Rosen, "Evaluation of ultrasonically determined elasticity-porosity relations in zinc oxide," Journal of the American Ceramic Society, vol. 79, no. 5, pp. 1281-1289, 1996.

[4] H. P. Schriemer, N. G. Pachet, and J. H. Page, "Ultrasonic investigation of the vibrational modes of a sintered glass-bead percolation system," Waves Random Media, vol. 6, no. 4, pp. 361-386, 1996.

[5] L. E. Kinsler, A. R. Frey, A. B. Coppens, and J. V. Sanders, Fundamentals of Acoustics, John Wiley \& Sons, New York, NY, USA, 4th edition, 2000.

[6] J. Kováčik, "Correlation between shear modulus and porosity in porous materials," Journal of Materials Science Letters, vol. 20, no. 21, pp. 1953-1955, 2001.

[7] J. Kováčik, "Correlation between Poisson's ratio and porosity in porous materials," Journal of Materials Science, vol. 41, no. 4, pp. 1247-1249, 2006.

[8] J. Kováčik, "Correlation between elastic modulus, shear modulus, poisson's ratio and porosity in porous materials," Advanced Engineering Materials, vol. 10, no. 3, pp. 250-252, 2008.

[9] R. W. Rice, "Use of normalized porosity in models for the porosity dependence of mechanical properties," Journal of Materials Science, vol. 40, no. 4, pp. 983-989, 2005.

[10] W. Pabst and E. Gregorová, "New relation for the porosity dependence of the effective tensile modulus of brittle materials," Journal of Materials Science, vol. 39, no. 10, pp. 3501-3503, 2004.

[11] http://en.wikipedia.org/wiki/Density_of_air. 

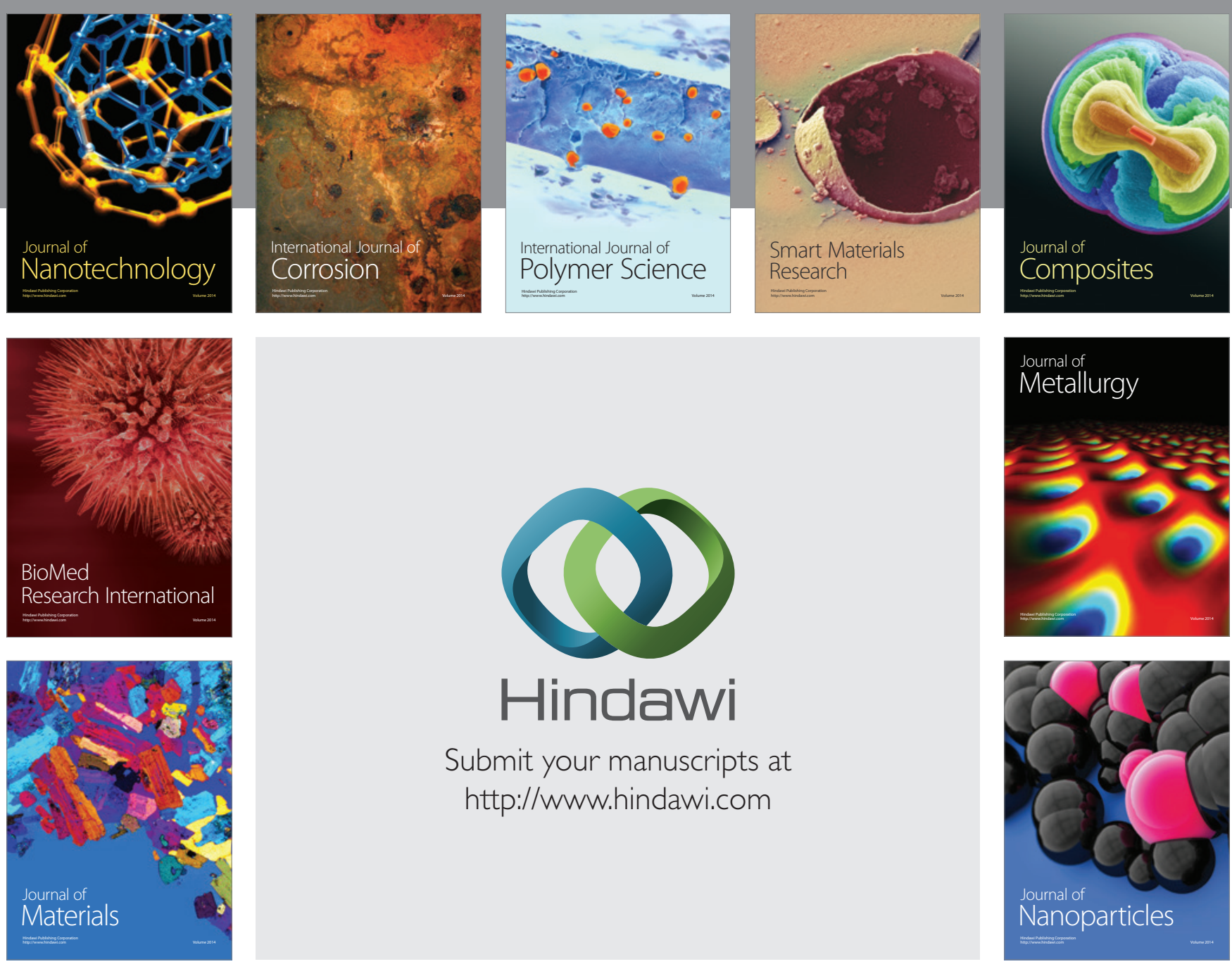

Submit your manuscripts at http://www.hindawi.com
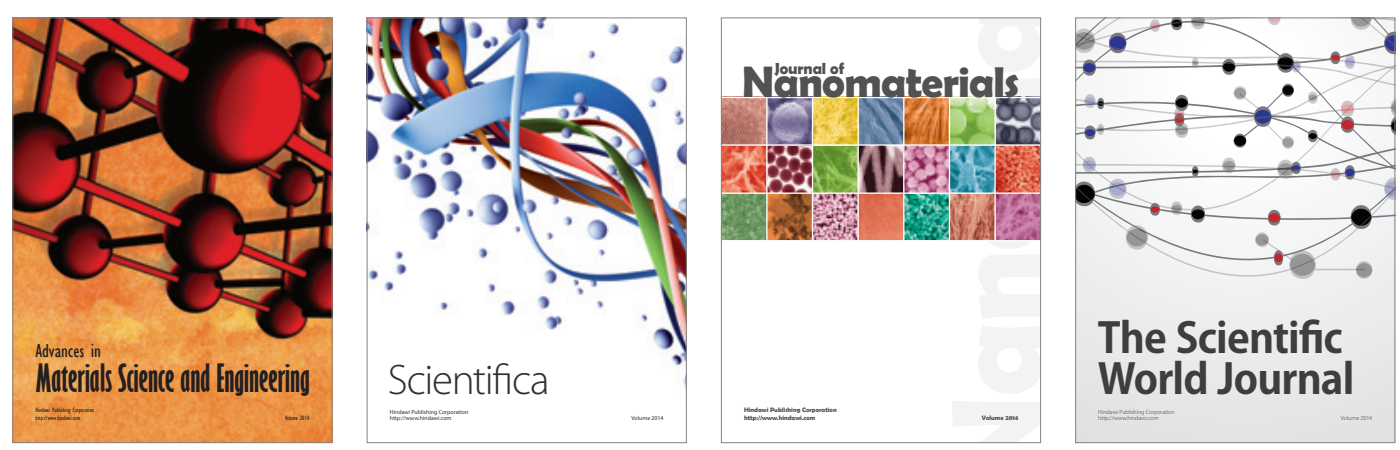

\section{The Scientific World Journal}
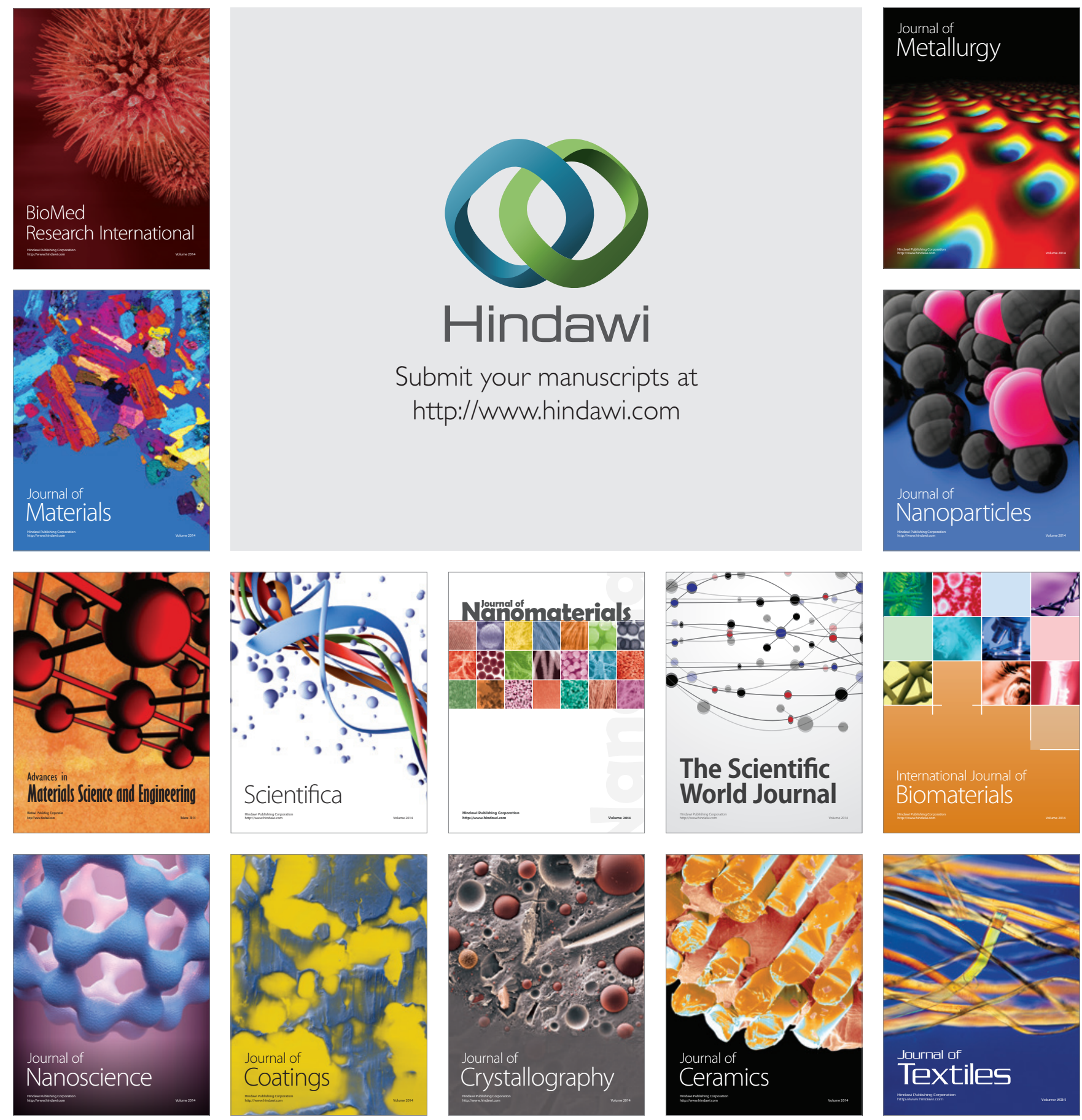